\title{
Modelling Pyramidal Absorbers Using the Fourier Modal Method and the Mode Matching Technique
}

\author{
Arya Fallahi, Associate Member, IEEE, and Amin Enayati, Member, IEEE,
}

\begin{abstract}
The problem of plane wave diffraction from the periodic structure of an infinite PEC wall lined with pyramidal absorbers is considered. A hybrid method based on the R-matrix Fourier modal method (RFMM) and the mode matching (MM) of fields is used for the efficient and robust analysis of this class of absorbers. The hybrid method benefits from the discrete spectrum of the periodic structure in transverse directions and consequently avoids spatial discretization along the periodicity axes. This leads to considerable reduction in the number of unknown coefficients. Furthermore, the method is capable of considering dispersive and inhomogeneous materials which are frequently used in the absorber industry. Several examples are outlined, simulated and measured to show the efficiency and accuracy of the method. The versatility and flexibility of the hybrid RFMM-MM technique makes it suitable for the optimal design of pyramidal absorbers to achieve further improvements in their performances.
\end{abstract}

Index Terms - Anechoic chambers, coupled mode analysis, electromagnetic diffraction, mode matching methods, numerical simulation, periodic structures, pyramidal absorbers, wedge absorbers.

\section{INTRODUCTION}

$\mathrm{E}$ LECTROMAGENTIC absorbers are by definition structures

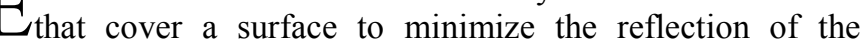
incident electromagnetic waves. Owing to their numerous applications in different domains from tracking technologies to electromagnetic compatibility (EMC), extensive research efforts in the past century are devoted to their analysis and design [1]. Generally, electromagnetic absorbers may be categorized into two main classes of $(i)$ resonating and $(i i)$ non-resonating structures.

In the first group, a lossy material is devised in form of a resonating structure. The electromagnetic resonance in conjunction with the loss results in substantial absorption of the incident wave. Several approaches are developed to realize the resonance in the lossy material, each of which leads to a particular absorber type. Some typical approaches are placing a resistive sheet in the distance of $\lambda_{0} / 4$ from a ground plane as in Salisbury screens [2, 3], using the Fabry-Perot resonances of a thin lossy layer [4] as well as multi-layers absorbing materials [5], the resonance frequencies of a periodic metallic array printed on a lossy material as in the metamaterial absorbers [6-12], and the resonance frequencies of periodically textured lossy material $[13,14]$. This absorber type offers a unique opportunity to fabricate flat configurations with portable geometries and low susceptibility to damage. Nonetheless, the main disadvantage is the narrowband operation due to the required resonance conditions.

Absorbers of the second type, i. e. non-resonating absorbers, function based on the gradual impinging of the incident wave into a lossy medium and losing of energy in the absorber material $[1,15,16]$. Pyramidal and wedge absorbers, widely used in anechoic chambers, are the most well-known type of such classes [17]. Their main benefit in comparison to the first type is the much larger operation bandwidth. Studying hybrid absorbers in which both types are properly combined to achieve best performing devices is a research topic focused by various groups. For this purpose, numerical techniques need to be developed that is capable of efficiently simulating both types as well as their combination. This paper is indeed one step towards achieving such a simulation tool.

In the last decades, a large number of numerical methods are proposed for the analysis of electromagnetic wave scattering by a periodic structure, which is the main problem in absorber simulation. The method of surface integral equation is developed for layered structures with homogeneous material properties [18, 19]. A more general version is solving the integral equation for polarization current, which removes the limitation to layered geometries [20]. The method of auxiliary sources is an efficient algorithm for two-dimensional structures with smooth surfaces [21]. Different algorithms based on the three dimensional discretization of the computational domain, like finitedifference time domain (FDTD) [22], finite element method (FEM) [23] and finite-difference frequency domain (FDFD) [24] are also tailored for the aforementioned problem. Their large computational cost compared to other approaches is the main drawback for using such methods for design and optimization.

The Fourier modal method (FMM) [25-28], sometimes referred to as rigorous coupled wave approach (RCWA) [29] or transmission line (TL) formulation [30], is a mature technique for solving the problem of plane wave scattering off a layered periodic structure. The combination of the method with mode matching technique for studying arbitrary geometries is also introduced in various studies [31, 32]. However, to the best of our knowledge, the method is never considered for solving the general problem of pyramidal absorbers in spite of its strong suitability. The main goals in this paper are presenting the simulation of pyramidal 


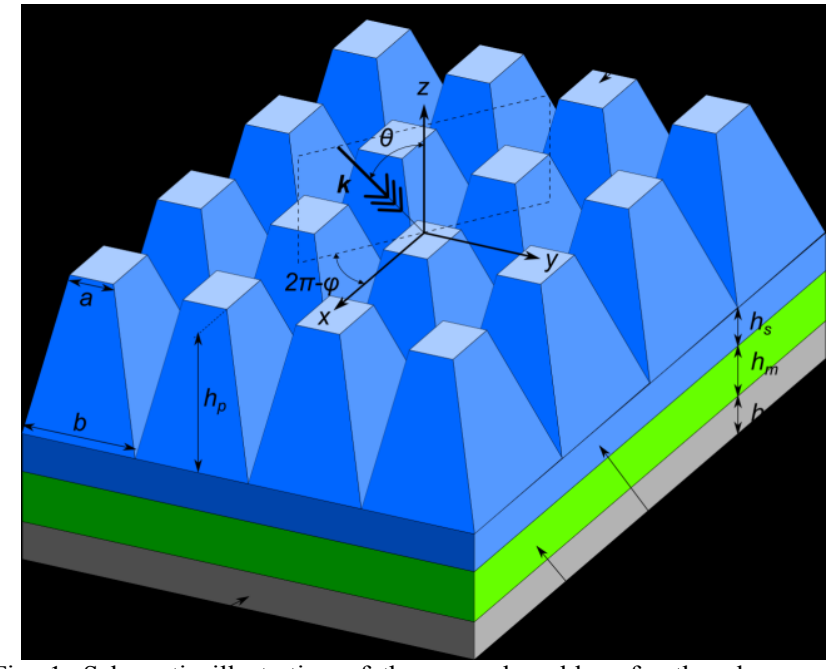

Fig. 1. Schematic illustration of the general problem for the plane wave scattering off hybrid pyramidal absorbers.

absorbers using the mode matching FMM algorithm and assessing its efficiency and accuracy through comparison with measurement results.

The paper is organized as follows: In Section II, the geometry and specification of the general considered problem is described. The methodology for solving the electromagnetic problem, meaning the R-matrix FMM method and its combination with mode matching, is thoroughly explained in Section III. Afterwards, in Section IV, several examples of pyramidal absorbers are analyzed, with results being compared against measurements. Finally, section V concludes the paper.

\section{PROBLEM SPECIFICATIONS}

Fig. 1 illustrates schematically the general geometry of hybrid pyramidal absorbers. The structure consists of arrays of vertical truncated pyramids arranged in a two dimensional lattice and placed on a multilayer configuration. Pyramids have two-fold symmetric properties along $x$ and $y$ axes, with heights denoted by $h_{p}$, top square side $a$ and bottom square side $b$. Generally, the multilayer structure under the pyramids may be made of three layers with different magnetic or electric properties: the substrate layer with height $h_{s}$, the matching layer with height $h_{m}$, and the ferrite layer with height $h_{f}$. The pyramids are made out of a lossy magnetic and dielectric material that could obtain inhomogeneous properties, meaning that the permittivity and permeability of the material could be functions of the vertical position, namely $\epsilon_{r}=\epsilon_{r}(z)$ and $\mu_{r}=\mu_{r}(z)$ with $0<z<h_{p}$. The whole structure is backed by a perfect electric conductor (PEC) surface on the lowest side. Note that, the general geometry shown in Fig. 1 is representative for hybrid absorbers mainly used for EMC applications incorporating truncated pyramids on top of Ferrite tiles. To be able to analyze microwave absorbers with sharp-tip pyramids, one can choose a, $h_{m}$, and $h_{f}$ equal to zero.

The goal of the analysis is the determination of the reflected electromagnetic fields when the structure is illuminated by a plane wave with a given angular frequency $\omega$, and direction $(\theta, \varphi)($ Fig. 1).

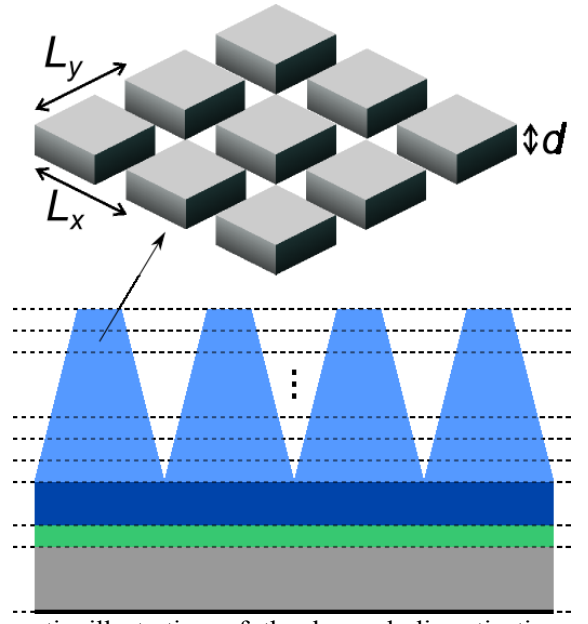

Fig. 2. Schematic illustration of the layered discretization used for the analysis of the pyramidal absorber and the basic configuration of each layer.

\section{Methodology}

To solve the described problem above, the structure geometry is firstly discretized into several thin layers, which is similar to the average medium technique [33, 34]. Afterwards, a concatenation of the layers forms the total geometry of the absorber. Fig. 2 shows the aforementioned discretization and the geometry of the basic layers analyzed for evaluating each of the pyramidal absorber. The procedure followed for solving the described problem above consists in two main parts: $(i)$ The mode matching technique to relate the incoming and outgoing fields of adjacent layers and (ii) the FMM or the TL technique to evaluate the R-matrix of each layer.

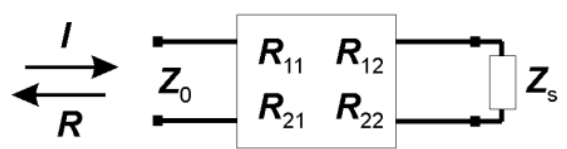

Fig. 3. R-matrix formulation; calculation of the total reflectivity of the pyramidal absorbers.

\section{A. Mode Matching Technique}

As mentioned before, the average medium technique evaluates each layer by considering a volumetric average permittivity and permeability for each layer. The method we use in this study calculates the properties of each layer using the FMM method providing a transmission line formulation for each layer, thereby maintaining the periodic nature of the layers. Subsequently, mode matching algorithm is used to concatenate the layers and acquire the total reflection coefficient.

By properties for each layer, the $\boldsymbol{R}$ matrix for each layer is meant, which is defined according to:

$$
\left(\begin{array}{l}
\boldsymbol{V}(d) \\
\boldsymbol{V}(0)
\end{array}\right)=\boldsymbol{R}\left(\begin{array}{l}
\boldsymbol{I}(d) \\
\boldsymbol{I}(0)
\end{array}\right)=\left(\begin{array}{ll}
\boldsymbol{r}_{11} & \boldsymbol{r}_{12} \\
\boldsymbol{r}_{21} & \boldsymbol{r}_{22}
\end{array}\right)\left(\begin{array}{l}
\boldsymbol{I}(d) \\
\boldsymbol{I}(0)
\end{array}\right)
$$

where $\boldsymbol{V}=\left(\boldsymbol{E}_{x}, \boldsymbol{E}_{y}\right)^{T}$ and $\boldsymbol{I}=\left(\boldsymbol{H}_{y},-\boldsymbol{H}_{x}\right)^{T}$. The vector $\boldsymbol{E}_{x}$ is defined as a vector containing all the electric field amplitudes of different diffraction orders along $x$ axis, i.e $E_{x m n}$. Once the $\boldsymbol{R}$ matrix is found for each layer the total reflection matrix of 
the absorber is calculated through the implementation of the following recursive equation:

$$
\begin{aligned}
& \boldsymbol{R}_{11}^{j}=\boldsymbol{r}_{11}^{j}+\boldsymbol{r}_{12}^{j} \boldsymbol{Z} \boldsymbol{r}_{21}^{j} \\
& \boldsymbol{R}_{12}^{j}=-\boldsymbol{r}_{12}^{j} \boldsymbol{Z} \boldsymbol{R}_{12}^{j-1} \\
& \boldsymbol{R}_{21}^{j}=\boldsymbol{R}_{21}^{j-1} \boldsymbol{Z} \boldsymbol{r}_{21}^{j} \\
& \boldsymbol{R}_{22}^{j}=\boldsymbol{R}_{22}^{j-1}-\boldsymbol{R}_{21}^{j-1} \boldsymbol{Z} \boldsymbol{R}_{12}^{j-1}
\end{aligned}
$$

with $\boldsymbol{Z}=\left(\boldsymbol{R}_{11}^{j-1}-\boldsymbol{r}_{22}^{j}\right)^{-1}$. In the above equations, $\boldsymbol{R}^{N}$, with $N$ the total number of layers, is the overall reflection matrix of the layers. Note that the $j$ index of each layer increases from top to bottom. In other words, $\boldsymbol{R}^{1}$ stands for the reflection matrix of the top layer with smallest amount of absorber material.

After acquiring the total $\boldsymbol{R}$ matrix of the layers, the whole tapered structure is replaced with an element characterized by the obtained $\boldsymbol{R}$ matrix, as shown in Fig. 3. Therefore, the reflection coefficient of each diffraction order is calculated from

$$
\begin{aligned}
& \boldsymbol{Z}_{t}=\boldsymbol{R}_{21}\left(\boldsymbol{Z}_{s}-\boldsymbol{R}_{11}\right)^{-1} \boldsymbol{R}_{12}+\boldsymbol{R}_{22}, \\
& \boldsymbol{R}_{t}=\left(\boldsymbol{Z}_{t}+\boldsymbol{Z}_{0}\right)^{-1}\left(\boldsymbol{Z}_{t}-\boldsymbol{Z}_{0}\right),
\end{aligned}
$$

where $\boldsymbol{Z}_{0}$ and $\boldsymbol{Z}_{s}$ are the impedance matrices of the material above the absorber and the equivalent impedance matrix seen from the top layer of the substrate, respectively.

To obtain $\boldsymbol{Z}_{s}$ matrix, the impedance matrix of each layer is required. The impedance matrix of a homogeneous layer is obtained from the following set of equations:

$$
\begin{aligned}
& Z=T\left(\begin{array}{cc}
Z^{T E} & 0 \\
0 & Z^{T M}
\end{array}\right) T^{T},
\end{aligned}
$$

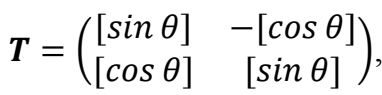

where $Z^{T E}$ and $Z^{T M}$ are diagonal matrices consisting in the intrinsic impedances of TE and TM modes $\left(Z_{m n}^{T E}=\omega \mu / k_{z_{m n}}\right.$ and $\left.Z_{m n}^{T M}=k_{z_{m n}} / \omega \epsilon\right)$, respectively. [ $\left.\sin \theta\right]$ and $[\cos \theta]$ are diagonal matrices of $\sin \theta_{m n}$ and $\cos \theta_{m n}$ with $\theta_{m n}=$ $\tan ^{-1} k_{y_{m n}} / k_{x_{m n}}$. Eventually, the propagation constants in different directions are obtained from:

$k_{z_{m n}}= \begin{cases}\sqrt{\epsilon \mu k_{0}^{2}-k_{x_{m n}}^{2}-k_{y_{m n}}^{2}} & \text { if } \epsilon \mu k_{0}^{2}>k_{x_{m n}}^{2}+k_{y_{m n}}^{2} \\ -j \sqrt{k_{x_{m n}}^{2}+k_{y_{m n}}^{2}-\epsilon \mu k_{0}^{2}} & \text { if } \epsilon \mu k_{0}^{2}<k_{x_{m n}}^{2}+k_{y_{m n}}^{2}\end{cases}$

where $k_{x_{m n}}=k_{x}+2 \pi m / L_{x}$ and $k_{y_{m n}}=k_{y}+2 \pi n / L_{y}$. The values $k_{x}$ and $k_{y}$ are the transverse propagation constants of the incident plane wave, and $L_{x}$ and $L_{y}$ are the lattice constants of the periodic structure along $x$ and $y$ axes.

There are different versions of mode matching and layer concatenation developed in the last decade. Some other examples are transmission matrix and $\boldsymbol{S}$ matrix approaches. The reason for selecting $\boldsymbol{R}$ matrix procedure lies in its superiority due to the numerically stable algorithm. For a detailed assessment of different algorithms the author is referred to [35].

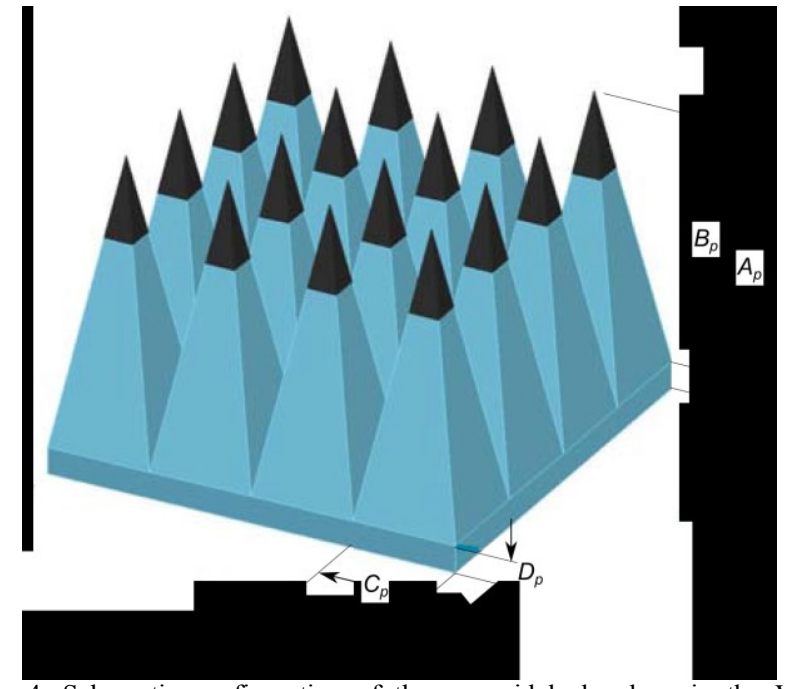

Fig. 4. Schematic configuration of the pyramidal absorbers in the VHP series.

\section{B. Fourier Modal Method}

The remaining step is how to obtain the $\boldsymbol{R}$ matrix for each layer. This is indeed where the transmission line (TL) modeling of a periodic structure is beneficial [30]. The geometry for which a TL formulation should be developed is illustrated in Fig. 2. The Bloch theorem requires that the source-free solutions to Maxwell's equations satisfy the following conditions:

$$
\left(\begin{array}{l}
\mathbf{E}(\mathbf{r}+\mathbf{L}) \\
\mathbf{H}(\mathbf{r}+\mathbf{L})
\end{array}\right)=\left(\begin{array}{c}
\mathbf{E}(\mathbf{r}) \\
\mathbf{H}(\mathbf{r})
\end{array}\right) e^{-j \mathbf{k} \cdot \mathbf{L}}
$$

which should hold at every position vector $\mathbf{r}=x \hat{\mathbf{x}}+y \hat{\mathbf{y}}+z \hat{\mathbf{z}}$. $\mathbf{L}=m \mathbf{a}_{1}+n \mathbf{a}_{2}$ is a vector connecting diagonal corners of the unit cell of the structure with $a_{1}=L_{x} \hat{x}$ and $a_{2}=L_{y} \hat{y}$ for a square lattice, and $\mathbf{k}=k_{x} \hat{\mathbf{x}}+k_{y} \hat{\mathbf{y}}$ denotes the transverse propagation vectors. We assume that the dielectric inclusion in each layer is made of a material with complex dielectric constant $\epsilon_{r}$. It is straightforward to prove that the following pseudo-Fourier series satisfy (7):

$$
\left(\begin{array}{l}
\mathbf{E}(\mathbf{r}+\mathbf{L}) \\
\mathbf{H}(\mathbf{r}+\mathbf{L})
\end{array}\right)=\sum_{m=-\infty}^{+\infty} \sum_{n=-\infty}^{+\infty}\left(\begin{array}{l}
\mathbf{E}_{m n}(z) \\
\mathbf{H}_{m n}(z)
\end{array}\right) e^{-j\left(\mathbf{k}+\mathbf{G}_{m n}\right) \cdot \mathbf{r}}
$$

where $\mathbf{G}_{m n}=m \mathbf{b}_{1}+n \mathbf{b}_{2}$ is a vector of the lattice reciprocal to the one defined by the vectors $\mathbf{L}_{m n}=m L_{x} \hat{x}+n L_{y} \hat{y}$. Note that for the unique characterization of the propagation mode, the coefficients $\mathbf{E}_{m n}(z)$ and $\mathbf{H}_{m n}(z)$ along with $k_{x}$ and $k_{y}$ should be determined.

As presented in [36], we define single column matrices $\boldsymbol{E}_{x}(z), \boldsymbol{E}_{y}(z), \boldsymbol{E}_{z}(z), \boldsymbol{H}_{x}(z), \boldsymbol{H}_{y}(z)$ and $\boldsymbol{H}_{z}(z)$ the entries of which are $E_{x_{m n}}(z), E_{y_{m n}}(z), E_{z_{m n}}(z), H_{x_{m n}}(z), H_{y_{m n}}(z)$ and $H_{z_{m n}}(z)$, respectively. From (8), one can easily deduce that $\partial / \partial x \equiv-j \boldsymbol{\alpha}$, and $\partial / \partial y \equiv-j \boldsymbol{\beta}$ with $\boldsymbol{\alpha}$ and $\boldsymbol{\beta}$ being diagonal matrices with diagonal elements equal to $\alpha_{m n}=k_{x}+m b_{1 x}+$ $n b_{2 x}$ and $\beta_{m n}=k_{y}+m b_{1 y}+n b_{2 y}$.

Now that the field quantities and their derivatives are expressed by matrices, let us derive an equivalent matrix expression for the constitutive relations. In this study, the 
pyramidal absorbers under investigation are assumed to be made of nonmagnetic, linear, isotropic dielectric materials. Under these assumptions the constitutive relations in the spatial domain are $\mathbf{B}(\mathbf{r})=\mu_{0} \mathbf{H}(\mathbf{r})$ and $\mathbf{D}(\mathbf{r})=\epsilon_{0} \epsilon(\mathbf{r}) \mathbf{E}(\mathbf{r})$. The relative permittivity in the single layer considered here is a biperiodic function of $x$ and $y$. Thus, $\epsilon(\mathbf{r})$ can be expanded into a pseudo-Fourier series with $z$-dependent expansion coefficients,

$$
\epsilon_{r}(\mathbf{r})=\sum_{m=-\infty}^{+\infty} \sum_{n=-\infty}^{+\infty} \epsilon_{m n}(z) e^{-j \mathbf{G}_{m n} \cdot \mathbf{r}}
$$

where the coefficients $\epsilon_{m n}(z)$ are obtained using the inverseFourier transform,

$$
\epsilon_{m n}(z)=\frac{1}{S} \iint_{S} \epsilon_{r}(\mathbf{r}) e^{-j \mathbf{G}_{m n} \cdot \mathbf{r}} .
$$

Once the expansion coefficients $\epsilon_{m n}(z)$ are determined, the relation $\mathbf{D}(\mathbf{r})=\epsilon_{0} \epsilon(\mathbf{r}) \mathbf{E}(\mathbf{r})$ can be expressed in matrix form as

$$
\boldsymbol{D}_{u}(z)=\epsilon_{0} \boldsymbol{\epsilon}(z) \boldsymbol{E}_{u}(z),
$$

where $u \in\{x, y, z\}$, and the matrices $\boldsymbol{D}_{u}(z)$ are defined in the same way as the matrices for the electric field components. The matrix $\boldsymbol{\epsilon}(z)$ is a $(2 M+1)(2 N+1)$ matrix with $M$ and $N$ being the Fourier truncation order in each direction and is obtained by the following relations

$$
\boldsymbol{\epsilon}(z)=\left(\begin{array}{cccc}
\boldsymbol{e}_{0} & \boldsymbol{e}_{-1} & \cdots & \boldsymbol{e}_{-2 M} \\
\boldsymbol{e}_{0} & \boldsymbol{e}_{0} & \cdots & \boldsymbol{e}_{-2 M+1} \\
\vdots & \vdots & \ddots & \vdots \\
\boldsymbol{e}_{2 M} & \boldsymbol{e}_{2 M-1} & \cdots & \boldsymbol{e}_{0}
\end{array}\right),
$$

with

$$
\boldsymbol{e}_{m}=\left(\begin{array}{cccc}
\epsilon_{m, 0} & \epsilon_{m,-1} & \cdots & \epsilon_{m,-2 N} \\
\epsilon_{m, 1} & \epsilon_{m, 0} & \cdots & \epsilon_{m,-2 N+1} \\
\vdots & \vdots & \ddots & \vdots \\
\epsilon_{m, 2 N} & \epsilon_{m, 2 N-1} & \cdots & \epsilon_{m, 0}
\end{array}\right)
$$

The constitutive relation $\mathbf{B}(\mathbf{r})=\mu_{0} \mathbf{H}(\mathbf{r})$ can be converted to the equivalent matrix equation

$$
\boldsymbol{B}_{u}(z)=\mu_{0} \mathbf{1}_{\boldsymbol{K} \times \boldsymbol{K}} \boldsymbol{H}_{u}(z),
$$

where $u \in\{x, y, z\}$, and the single-column matrices $\boldsymbol{B}_{u}(z)$ contain the expansion coefficients of $\boldsymbol{B}(\mathbf{r})$ which are sorted like the previous matrices for field quantities.

Substituting the proposed solutions given by (8) along with the constitutive relations (11) and (14) in Maxwell's equations and eliminating the matrices of field components perpendicular to the grating surface $\left(\boldsymbol{E}_{z}\right.$ and $\left.\boldsymbol{H}_{z}\right)$ leads to the desired TL formulation

$$
\begin{gathered}
\frac{d}{d z}\left(\begin{array}{c}
\boldsymbol{E}_{x} \\
\boldsymbol{E}_{y}
\end{array}\right)=-j \omega \boldsymbol{L}\left(\begin{array}{c}
\boldsymbol{H}_{y} \\
-\boldsymbol{H}_{x}
\end{array}\right) \\
\frac{d}{d z}\left(\begin{array}{c}
\boldsymbol{H}_{y} \\
-\boldsymbol{H}_{x}
\end{array}\right)=-j \omega \boldsymbol{L}\left(\begin{array}{c}
\boldsymbol{E}_{x} \\
\boldsymbol{E}_{y}
\end{array}\right)
\end{gathered}
$$

where the matrices $\boldsymbol{L}$ and $\boldsymbol{C}$ are of $2 K \times 2 K$ dimension, and are given by

$$
\begin{gathered}
\boldsymbol{L}=\mu_{0}\left(\begin{array}{cc}
\mathbf{1}-\overline{\boldsymbol{\alpha}} \boldsymbol{\epsilon}^{-1} \overline{\boldsymbol{\alpha}} & -\overline{\boldsymbol{\alpha}} \boldsymbol{\epsilon}^{-1} \overline{\boldsymbol{\beta}} \\
-\overline{\boldsymbol{\beta}} \boldsymbol{\epsilon}^{-1} \overline{\boldsymbol{\alpha}} & \mathbf{1}-\overline{\boldsymbol{\beta}} \boldsymbol{\epsilon}^{-1} \overline{\boldsymbol{\beta}}
\end{array}\right) \\
\boldsymbol{C}=\epsilon_{0}\left(\begin{array}{cc}
\boldsymbol{\epsilon}-\overline{\boldsymbol{\beta}}^{2} & -\overline{\boldsymbol{\beta}} \overline{\boldsymbol{\alpha}} \\
\overline{\boldsymbol{\alpha}} \overline{\boldsymbol{\beta}} & \boldsymbol{\epsilon}-\overline{\boldsymbol{\alpha}}^{2}
\end{array}\right)
\end{gathered}
$$

where $\mathbf{1}=\mathbf{1}_{K \times K}, \overline{\boldsymbol{\alpha}}=\boldsymbol{\alpha} / k_{0}$, and $\overline{\boldsymbol{\beta}}=\boldsymbol{\beta} / k_{0}$, with $k_{0}=$ $\omega \sqrt{\epsilon_{0} \mu_{0}}$.

Using the developed telegraph equations, the single layer structure shown in Fig. 2 is to be analyzed. The periodic region has a finite height which causes the electromagnetic fields to vary along the $z$-direction. The structure is often referred to as a dielectric photonic crystal slab. The periodic relative permittivity is expandable in a Fourier series of the form (9). In addition, the telegraph equation given by (15) can be used to determine the natural modes of propagation. To this end, the equivalent voltage and current vectors are defined as $\boldsymbol{V}=\left(\boldsymbol{E}_{x}, \boldsymbol{E}_{y}\right)^{T}$ and $\boldsymbol{I}=\left(\boldsymbol{H}_{y},-\boldsymbol{H}_{x}\right)^{T}$. Therefore, the telegraph equation is transformed to a set of coupled-wave equations as

$$
\frac{d}{d z}\left(\begin{array}{l}
\boldsymbol{V}(z) \\
\boldsymbol{I}(z)
\end{array}\right)=-j \omega\left(\begin{array}{ll}
\mathbf{0} & \boldsymbol{L} \\
\boldsymbol{C} & \mathbf{0}
\end{array}\right)\left(\begin{array}{c}
\boldsymbol{V}(z) \\
\boldsymbol{I}(z)
\end{array}\right) .
$$

It can be easily shown that the $4 K \times 4 K$ system of equations (17) may be reduced to

$$
\frac{d^{2} \boldsymbol{V}}{d z^{2}}=-\omega^{2} \boldsymbol{L} \boldsymbol{C} \boldsymbol{V} .
$$

The equivalent voltage and current vectors in the periodic structure is expressed through eigenvectors $\boldsymbol{P}$ and $\boldsymbol{Q}$, and eigenvalues $k_{z}$ as

$$
\left(\begin{array}{c}
\boldsymbol{V}(z) \\
\boldsymbol{I}(z)
\end{array}\right)=\left(\begin{array}{cc}
\boldsymbol{P} \boldsymbol{X}^{+} & \boldsymbol{P} \boldsymbol{X}^{-} \\
\boldsymbol{Q} \boldsymbol{X}^{+} & -\boldsymbol{Q} \boldsymbol{X}^{-}
\end{array}\right)\left(\begin{array}{l}
\boldsymbol{c}^{+} \\
\boldsymbol{c}^{-}
\end{array}\right)
$$

where $\boldsymbol{c}^{+}$and $\boldsymbol{c}^{-}$are the amplitude vectors of forward and backward waves and $\boldsymbol{X}^{ \pm}=\boldsymbol{e}^{\mp \boldsymbol{j} \boldsymbol{k}_{\mathrm{Z}} \boldsymbol{Z}}$ are diagonal matrices with elements equal to $e^{\mp j k_{z n} z}$. $\boldsymbol{P}$ is obtained from the eigenvectors of $\omega^{2} \boldsymbol{L C}$ and $k_{z n}=\sqrt{\lambda_{n}}$, where $\lambda_{n}$ is the eigenvalue of the same matrix. $\boldsymbol{Q}$ is obtained through $Q=\omega \boldsymbol{C P} \boldsymbol{k}_{z}^{-1}$, where $\boldsymbol{k}_{z}$ is the assumed to be a diagonal matrix with its diagonal elements being equal to $k_{z n}$.

Using the equation (8) for the propagating voltage and current the $\boldsymbol{R}$ matrix of a layer can be found as the following:

$$
\begin{aligned}
& \boldsymbol{r}_{11}^{j}=j \boldsymbol{P}\left(\boldsymbol{Q} \tan \boldsymbol{k}_{\mathbf{z}} d\right)^{-1} \\
& \boldsymbol{r}_{12}^{j}=-j \boldsymbol{P}\left(\boldsymbol{Q} \sin \boldsymbol{k}_{\mathbf{z}} d\right)^{-1} \\
& \boldsymbol{r}_{21}^{j}=j \boldsymbol{P}\left(\boldsymbol{Q} \sin \boldsymbol{k}_{\boldsymbol{z}} d\right)^{-1} \\
& \boldsymbol{r}_{22}^{j}=-j \boldsymbol{P}\left(\boldsymbol{Q} \tan \boldsymbol{k}_{\mathbf{z}} d\right)^{-1}
\end{aligned}
$$

The above listed equations in conjunction with the pertinent TL formulation to obtain the matrices $\boldsymbol{P}, \boldsymbol{Q}$ and $\boldsymbol{k}_{\boldsymbol{z}}$ offers a complete set of equations to solve any periodic multilayer structure, including the periodic structure of wedge and pyramidal absorbers. In the next section, we use the developed software based on the introduced formulation for modeling the considered absorbers. 


\section{APPLICATION EXAMPLES}

In this section, we outline some practical pyramidal absorbers and try to simulate them using the proposed method. The results are then compared against measurement.

\section{A. ECCOSORB $\mathrm{VHP}-4$}

The first studied pyramidal absorber is the ECCOSORB VHP-4 absorber. The basic configuration of this absorber is shown in Fig. 4, where the dimensions are measured as $A_{p}=10.2 \mathrm{~cm}, B_{p}=7.7 \mathrm{~cm}, C_{p}=3.8 \mathrm{~cm}$, and $D_{p}=2.5 \mathrm{~cm}$. To obtain the absorber characteristics over a wide frequency bandwidth different measurement techniques are utilized, namely ARCH, COAX, WG300, WG500 and WG1000. Each of these techniques measures the reflectivity of the absorbers under test over a certain frequency band. The Arch setup which is composed of two antennas looking into the absorber under test is the most standard measurement technique to measure the reflectivity of absorbers [37]. After calibrating the setup, the reflectivity of the absorbers are measured in the frequency band of 2-6 GHz. In order to have the incident wave on the absorbers under test similar to plane waves, they should be placed in the far-field of the antennas. Moreover, the lateral size of the absorber under test should be large enough to reduce the edge effects. Hence, for the lower frequencies, e. g. bellow $1 \mathrm{GHz}$, the size of the arch setup as well as the number of absorbers needed to be measured becomes so huge that guided-wave structures with close boundaries are used for the reflectivity measurements instead of the Arch setup. To cover the frequencies from $10 \mathrm{MHz}$ to $1.2 \mathrm{GHz}$, reported here as the measurement results, 4 different setups have been used. These 4 setups can be divided into 2 main groups of coaxial and hollow waveguides. The Coaxial waveguide [38], named as COAX, is capable of measuring the reflectivity of the absorbers under test from very low frequencies, e. g. $10 \mathrm{MHz}$, up to $140 \mathrm{MHz}$ which is the close to its second-mode cut-off frequency. For the rest of the frequencies, i. e. 140-320 MHz, 380-480 MHz, and 700-1200 MHz, WG300, WG500 and WG1000 are used respectively. Note that these 3 setups are shorted hollow metallic waveguides [39] in which the absorbers under test are placed for reflectivity measurement. In Table 1, the different measurement methods are listed with the corresponding frequency bandwidth.

\begin{tabular}{|c|c|}
\hline COAX & $10 \mathrm{MHz}-140 \mathrm{MHz}$ \\
\hline WG300 & $140 \mathrm{MHz}-320 \mathrm{MHz}$ \\
\hline WG500 & $380 \mathrm{MHz}-480 \mathrm{MHz}$ \\
\hline WG1000 & $700 \mathrm{MHz}-1.2 \mathrm{GHz}$ \\
\hline ARCH & $2 \mathrm{GHz}-6 \mathrm{GHz}$ \\
\hline
\end{tabular}

Table 1. Different measurement setups used for the absorbers and their corresponding frequency interval.

The reflectivity of the ECCOSORB VHP-4 absorber under normal incidence is simulated by the RFMM method. For the

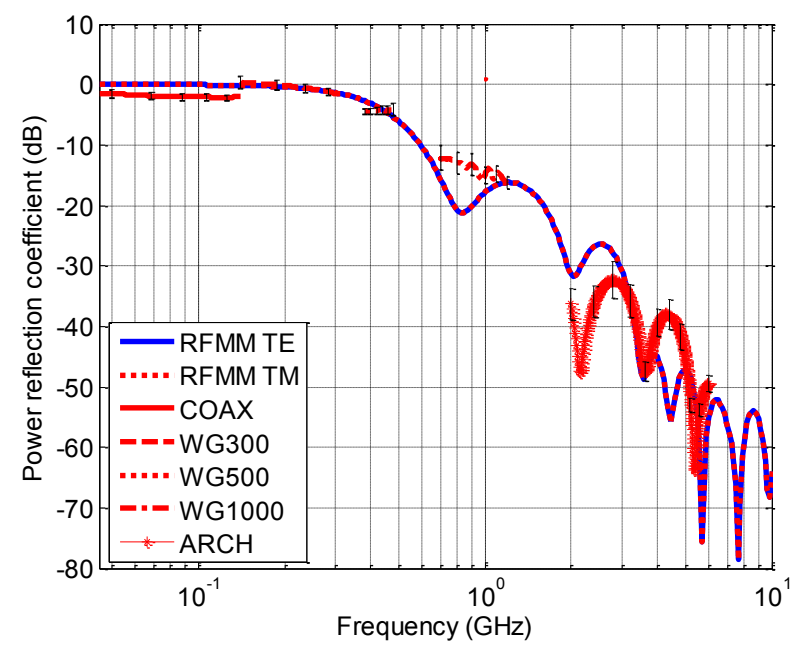

Fig. 5. Reflectivity of the ECCOSORB VHP-4 in the normal plane-wave incidence obtained using the RFMM model and compared against measured values. The error bars are based on the uncertainty measures in the experiment setup.
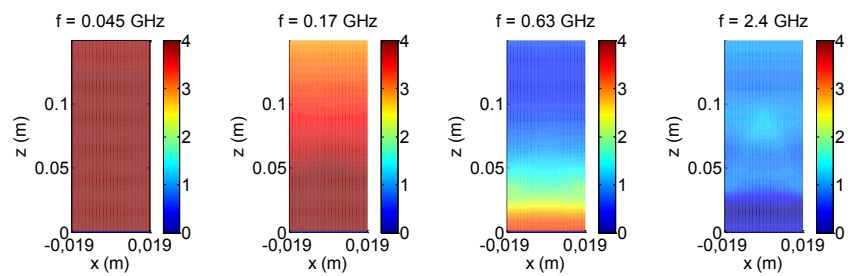

Fig. 6. Power profile (normalized to the power of the incident beam) of the ECCOSORB VHP-4 simulated using the RFMM method at four different frequency points.

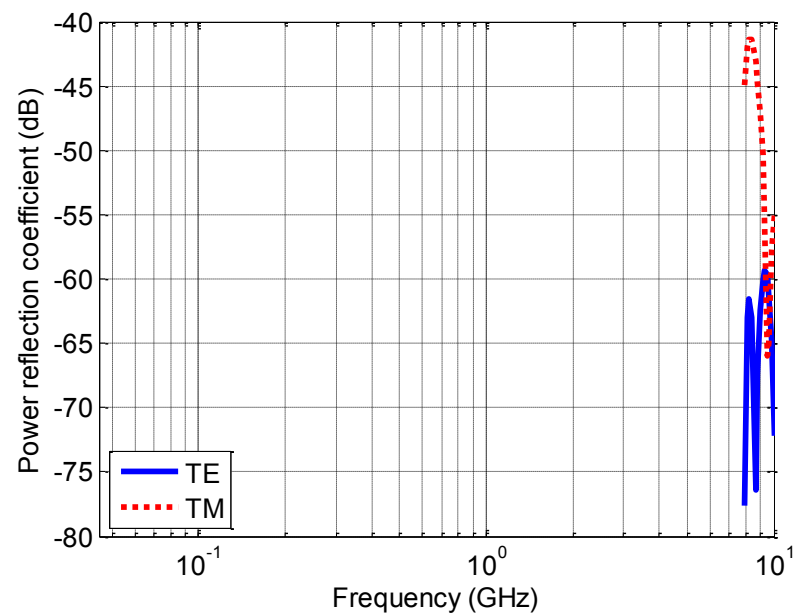

Fig. 7. Reflected energy versus frequency for the first order Flouquet mode obtained using RFMM method. The cut-off frequency of the mode is 7.9 $\mathrm{GHz}$.

simulation, the permittivity of the absorbing material needs to be measured. A wideband coaxial setup has been used to extract the material characteristics of different absorbers investigated. A TRL calibration followed by an iterative postprocessing technique is used to extract the permittivity and permeability of different absorber samples [40]. 


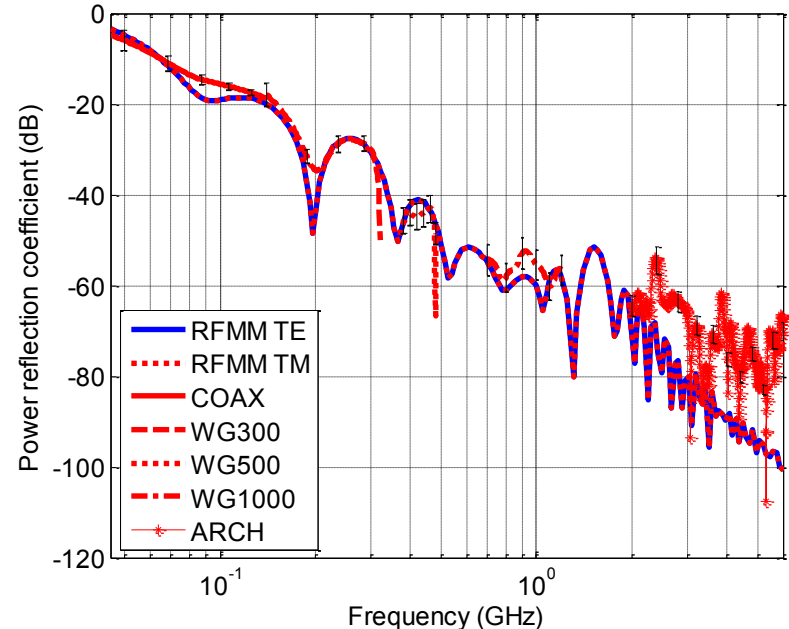

Fig. 8. Reflectivity of the ECCOSORB VHP-36 in the normal plane-wave incidence obtained using the RFMM model and compared against measured values. The error bars are based on the uncertainty measures in the experiment setup.

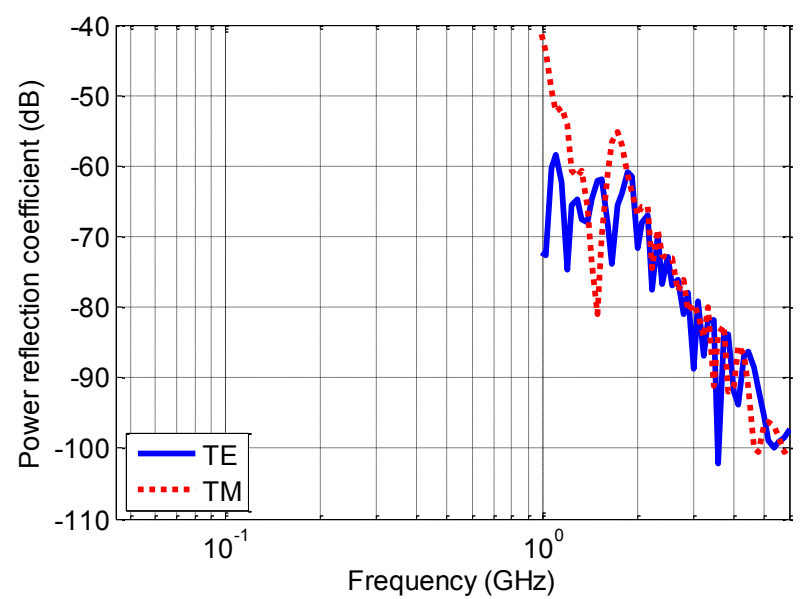

Fig. 9. Reflected energy versus frequency for the first order Flouquet mode obtained using the RFMM method. The cut-off frequency of the mode is 0.98 $\mathrm{GHz}$.

Based on the measured data, the relative permittivity of the material is fitted to a exponential function which is subsequently used in the RFMM method for evaluating the absorption performance. The simulation results obtained from RFMM method are shown in Fig. 5. The RFMM method is coded in MATLAB and run on a PC with INTEL i5 core and 8 GB of RAM. The simulation speed is very much dependent on the Fourier truncation order and the number of layers assumed for the discretization of the pyramidal region. For a simulation with $M=N=5$ as the Fourier truncation order and 100 layers for the pyramidal region, the calculation in each frequency takes about 4 seconds. These numbers are chosen after investigating the convergence of the results. It means that increasing the truncation order or the layer discretization does not introduce a considerable change in the simulation results. The simulation result is also compared against the measured values, which evidences the reliability of the developed algorithm. In order to illustrate the performance of the absorber, we visualize the electromagnetic energy profile on a plane formed by the polarization vector and the pyramid axis.

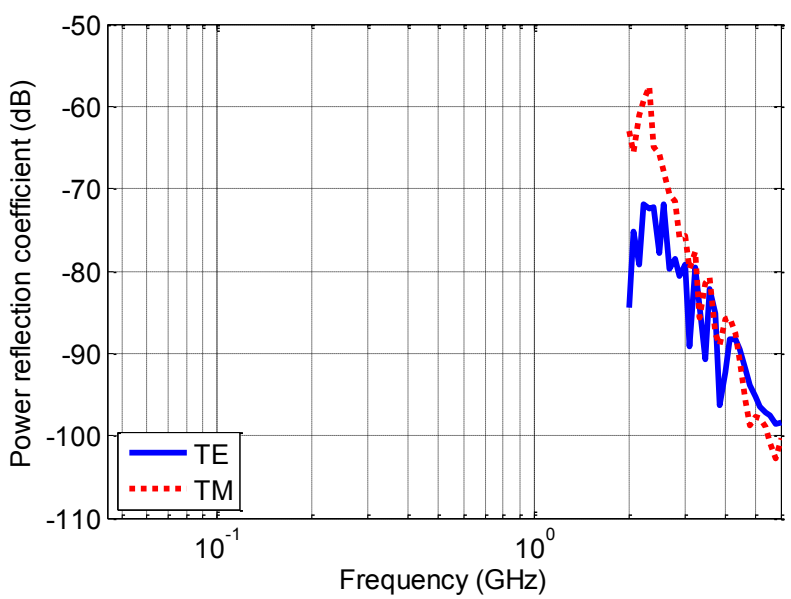

Fig. 10. Reflected energy versus frequency for the second order Flouquet mode obtained using RFMM method. The cut-off frequency of the mode is $1.97 \mathrm{GHz}$.

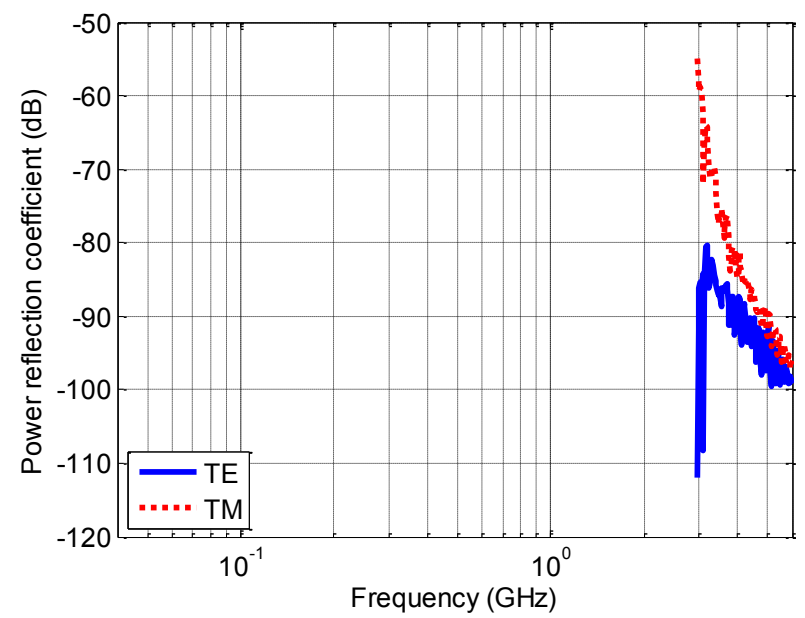

Fig. 11. Reflected energy versus frequency for the third order Flouquet mode obtained using RFMM method. The cut-off frequency of the mode is 2.95 $\mathrm{GHz}$.

The energy profiles in different frequency points are shown in Fig. 6. The absorbing behavior of the field in higher frequencies is evident in the visualizations.

One of the promising features of the RFMM method is its ability to evaluate the reflected power in all directions. In other words, the amplitude of all the Flouquet harmonics are obtained in one single simulation. For the considered example, the cut-off frequency of the second order Flouquet harmonic is about $7.9 \mathrm{GHz}$, meaning that for the frequencies higher than this value some of the incident power is reflected with certain reflection angle dependent on the frequency. The amplitude of the second reflected Flouquet mode in terms of the frequency is depicted in Fig. 7.

\section{B. ECCOSORB VHP-36}

ECCOSORB VHP-36 is the second absorber simulated to verify the developed model. The configuration of this absorber is similar to VHP-4, shown in Fig. 4. The dimensions are $A_{p}=91.4 \mathrm{~cm}, B_{p}=76.4 \mathrm{~cm}, C_{p}=30.5 \mathrm{~cm}, \quad$ and $D_{p}=$ $15 \mathrm{~cm}$. Again, the reflectivity of the absorber is measured in different frequency bands using the aforementioned techniques. The fitting of the measured permittivity of the 
absorbing material to an exponential function is used to evaluate the absorption performance with RFMM software. Fig. 8 presents the simulated results for the ECCOSORB VHP-36 absorber compared with the measured values. A good agreement between the simulation and measurement results is observed.

Due to the longer lattice constant in VHP-36 absorber compared to the VHP-4 case, the cut-off frequency for the higher order Flouquet modes are smaller. This results in the propagation of several Flouquet modes in high frequencies. In the considered frequency interval, three higher order modes are able to propagate, whose power reflection coefficients are depicted in terms of frequency in Fig. 9-11. The cut-off frequencies for the first, second and third higher order modes are $0.98 \mathrm{GHz}, 1.97 \mathrm{GHz}$, and $2.95 \mathrm{GHz}$, respectively.

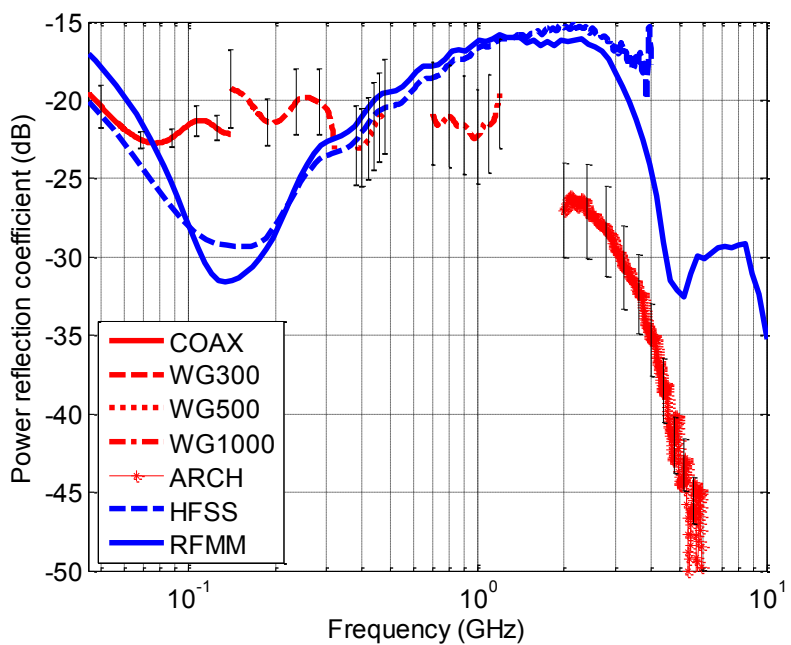

Fig. 12. Reflectivity of the ECCOSORB VHY-30 in the normal plane-wave incidence obtained using the RFMM model and compared against measured values. The error bars are based on the uncertainty measures in the experiment setup.

\section{ECCOSORB VHY-30}

The last absorber considered for the verification of the developed RFMM code is the ECCOSORB VHY-30 absorber. The absorber configuration corresponds to the general structure shown in Fig. 1. The first layer consisting of the truncated pyramids has the dimensions $b=30 \mathrm{~cm}, a=$ $8.2 \mathrm{~cm}$, and $h_{p}=69.2 \mathrm{~cm}$. Underneath the pyramids resides a substrate layer with the thickness $h_{s}=8.2 \mathrm{~cm}$ and is made out of the same absorbing material as the pyramids. The matching layer with thickness $h_{m}=1 \mathrm{~cm}$ is made out of foam which has material properties close to air. The lowermost layer is the ferrite layer with thickness $h_{f}=6 \mathrm{~mm}$. Similar to the previous examples, the material properties of the pyramid and the substrate are modelled and given to the RFMM code. We have also performed measurement techniques to characterize the ferrite layer for calculating the overall reflection of the absorber VHY-30.

We use the illustrated material properties and the given dimensions to calculate the absorption properties of the VHY30 absorber. The results are shown in Fig. 12 and compared against measurements. The strong absorption of this multilayer absorber dictates measurement of very minute

signals in the reflection spectrum, which is in long wavelengths very challenging. Additionally, in the low wavelength regime, a reliable measurement requires accurate consideration of the diffraction effects and the radiation pattern of the utilized antenna. Fulfilling these tasks with a high accuracy in the level of $-30 \mathrm{~dB}$ to $-50 \mathrm{~dB}$ is very difficult in an ARCH measurement setup. For this reason, achieving a general good agreement with the simulation results is very cumbersome. We have additionally evaluated the absorber reflection using the commercial software Ansoft HFSS which solves the problem using a finite element method (FEM) in order to better verify the RFMM simulation method. The agreement between the two different simulation techniques shows the promise of our semi-analytical approach. There are of course some discrepancies in the results in the tails of the considered frequency range for the FEM simulation. This is indeed a well-known effect. In the long wavelengths the inaccurate mesh truncation reduces the calculation precision and in the high frequency (short wavelength) range the improper discretization leads to the same difficulty.

\section{CONCLUSION}

A hybrid method is presented for the efficient analysis of the pyramidal absorbers. The method takes advantage of the Fourier Modal Method (FMM) to consider the periodicity of the structure in the both transverse dimensions. The R-matrix method in conjunction with a planar discretization of the pyramidal region results in a rigorous and robust technique for modelling pyramidal absorbers. Various absorbers are simulated using this method and compared with measurement results. We believe the introduced technique will be very advantageous for future assessment of multilayer absorbers as well as the metamaterial absorbers.

\section{REFERENCES}

[10]
W. Emerson, "Electromagnetic wave absorbers and anechoic chambers through the years," Antennas and Propagation, IEEE Transactions on, vol. 21, pp. 484-490, 1973.

R. L. Fante and M. T. Mccormack, "Reflection properties of the Salisbury screen," Antennas and Propagation, IEEE Transactions on, vol. 36, pp. 1443-1454, 1988.

W. W. Salisbury, "Absorbent Body for Electromagnetic Waves," 1952.

X. Yu, G. Lin, D. Zhang, and H. He, "An optimizing method for design of microwave absorbing materials," Materials \& design, vol. 27, pp. 700-705, 2006. Propagation Magazine, IEEE, vol. 36, pp. 17-25, 1994.

N. Engheta, "Thin absorbing screens using metamaterial surfaces," in Antennas and Propagation Society International Symposium, 2002. IEEE, 2002, pp. 392-395.

D. Kern and D. Werner, "A genetic algorithm approach to the design of ultra-thin electromagnetic bandgap absorbers," Microwave and Optical Technology Letters, vol. 38, pp. 61-64, 2003.

Q. Gao, Y. Yin, D.-B. Yan, and N.-C. Yuan, "Application of metamaterials to ultra-thin radar-absorbing material design," Electronics Letters, vol. 41, pp. 936-937, 2005.

H. Mosallaei and K. Sarabandi, "A one-layer ultra-thin metasurface absorber," in Antennas and Propagation Society International Symposium, 2005 IEEE, 2005, pp. 615-618.

A. K. Zadeh and A. Karlsson, "Capacitive circuit method for fast and efficient design of wideband radar absorbers," Antennas and Propagation, IEEE Transactions on, vol. 57, pp. 2307-2314, 2009. 
[11] F. Costa, A. Monorchio, and G. Manara, "Analysis and design of ultra thin electromagnetic absorbers comprising resistively loaded high impedance surfaces," Antennas and Propagation, IEEE Transactions on, vol. 58, pp. 1551-1558, 2010.

[12] H.-Y. Chen, H.-B. Zhang, and L.-J. Deng, "Design of an ultra-thin magnetic-type radar absorber embedded with FSS," Antennas and Wireless Propagation Letters, IEEE, vol. 9, pp. 899-901, 2010.

[13] A. Fallahi, A. Yahaghi, H. Benedickter, H. Abiri, M. Shahabadi, and C. Hafner, "Thin wideband radar absorbers," Antennas and Propagation, IEEE Transactions on, vol. 58, pp. 4051-4058, 2010.

[14] A. Fallahi, M. Mishrikey, C. Hafner, and R. Vahldieck, "Radar absorbers based on frequency selective surfaces on perforated substrates," Journal of Computational and Theoretical Nanoscience, vol. 5, pp. 704-710, 2008.

[15] O. M. Bucci and G. Franceschetti, "Scattering from wedge-tapered absorbers," Antennas and Propagation, IEEE Transactions on, vol. 19, pp. 96-104, 1971.

[16] B. T. Dewitt and W. D. Burnside, "Electromagnetic scattering by pyramidal and wedge absorber," Antennas and Propagation, IEEE Transactions on, vol. 36, pp. 971-984, 1988.

[17] B. T. DeWitt and W. D. Burnside, "Analysis and measurement of electromagnetic scattering by pyramidal and wedge absorbers," NASA STI/Recon Technical Report N, vol. 87, p. 27873, 1986.

[18] R. Janaswamy, "Oblique scattering from lossy periodic surfaces with applications to anechoic chamber absorbers," Antennas and Propagation, IEEE Transactions on, vol. 40, pp. 162-169, 1992.

[19] B. Gallinet, A. M. Kern, and O. J. Martin, "Accurate and versatile modeling of electromagnetic scattering on periodic nanostructures with a surface integral approach," JOSA A, vol. 27, pp. 2261-2271, 2010.

[20] C.-F. Yang, W. D. Burnside, and R. C. Rudduck, "A doubly periodic moment method solution for the analysis and design of an absorber covered wall," Antennas and Propagation, IEEE Transactions on, vol. 41, pp. 600-609, 1993.

[21] K. Tavzarashvili, C. Hafner, C. Xudong, R. Vahldieck, D. Kakulia, G. Ghvedashvili, et al., "Method of auxiliary sources and modelbased parameter estimation for the computation of periodic structures," Journal of Computational and Theoretical Nanoscience, vol. 4, pp. 667-674, 2007.

[22] A. Taflove and S. C. Hagness, "Computationai Electrodynamics," 2000 .

[23] Z. Chen and H. Wu, "An adaptive finite element method with perfectly matched absorbing layers for the wave scattering by periodic structures," SIAM Journal on Numerical Analysis, vol. 41, pp. 799-826, 2003.

[24] W. Sun, K. Liu, and C. A. Balanis, "Analysis of singly and doubly periodic absorbers by frequency-domain finite-difference method," Antennas and Propagation, IEEE Transactions on, vol. 44, pp. 798-805, 1996.

[25] L. Li, "Fourier modal method for crossed anisotropic gratings with arbitrary permittivity and permeability tensors," Journal of Optics A: Pure and Applied Optics, vol. 5, p. 345, 2003.

[26] L. Li, "Fourier Modal Method," Gratings: Theory and Numeric Applications, Second Revisited Edition, 2014.

[27] L. Li, "New formulation of the Fourier modal method for crossed surface-relief gratings," JOSA A, vol. 14, pp. 2758-2767, 1997.

[28] P. Lalanne and E. Silberstein, "Fourier-modal methods applied to waveguide computational problems," Optics Letters, vol. 25, pp. 1092-1094, 2000.

[29] M. Moharam and T. Gaylord, "Rigorous coupled-wave analysis of planar-grating diffraction," JOSA, vol. 71, pp. 811-818, 1981.

[30] A. Fallahi, K. Z. Aghaie, A. Enayati, and M. Shahabadi, "Diffraction analysis of periodic structures using a transmissionline formulation: principles and applications," Journal of Computational and Theoretical Nanoscience, vol. 4, pp. 649-666, 2007.

[31] L. Li, "Multilayer modal method for diffraction gratings of arbitrary profile, depth, and permittivity," JOSA A, vol. 10, pp. 2581-2591, 1993.

[32] E. Popov, M. Neviere, B. Gralak, and G. Tayeb, "Staircase approximation validity for arbitrary-shaped gratings," JOSA A, vol. 19, pp. 33-42, 2002.

[33] E. F. Kuester and C. L. Holloway, "A low-frequency model for wedge or pyramid absorber arrays-I: Theory," Electromagnetic Compatibility, IEEE Transactions on, vol. 36, pp. 300-306, 1994.
[34] C. L. Holloway and E. F. Kuester, "A low-frequency model for wedge or pyramid absorber arrays-II: Computed and measured results," Electromagnetic Compatibility, IEEE Transactions on, vol. 36, pp. 307-313, 1994.

[35] L. Li, "Formulation and comparison of two recursive matrix algorithms for modeling layered diffraction gratings," JOSA A, vol. 13, pp. 1024-1035, 1996.

[36] M. Shahabadi, S. Atakaramians, and N. Hojjat, "Transmission line formulation for the full-wave analysis of two-dimensional dielectric photonic crystals," in Science, Measurement and Technology, IEE Proceedings-, 2004, pp. 327-334.

[37] H. Peus, " Error-corrected wideband reflectivity measurement of microwave absorbing materials using the arch technique," Mikrowellen Magzine, vol. 14, pp. 524-526, Sept. 1988.

[38] H. Peus, "Electromagnetic absorber measurement in a large coax," in Proc. $9^{\text {th }}$ Int. Zurich Symp. on EMC, 1991, pp. 541-546.

[39] H. Peus, "Electromagnetic absorber measurement in a large waveguiode," in Proc. $8^{\text {th }}$ Int. Zurich Symp. on EMC, March 1989, pp. 253-258.

[40] J. Baker-Jarvis, M. D. Janezic, J. H. Grosvenor, and R. G. Geyer, "Transmission/reflection and short-circuit line methods for measuring permittivity and permeability," NIST Technical Note $1355,1992$. 